\title{
INTEGRATED DIRECT-MODULATION BASED QUANTUM CRYPTOGRAPHY SYSTEM
}

\author{
Johann Cussey ${ }^{1}$, Matthieu Bloch ${ }^{2}$, Jean-Marc Merolla' ${ }^{1}$ and Steven.W McLaughlin ${ }^{2}$ \\ ${ }^{1}$ GTL-CNRS Telecom UMR 6174, 2-3 rue Marconi 57070 Metz, France, \\ jcussey@georgiatech-metz.fr \\ ${ }^{2}$ Electrical and Computer Engineering, Georgia Institute of Technology, Atlanta, Georgia, \\ USA, \\ mbloch@georgiatech-metz.fr
}

\begin{abstract}
We report a new quantum key distribution scheme using direct-modulation method associated with single sideband detection (SSB). Experiments were carried out at $850 \mathrm{~nm}$ using standard electronic and optical components.
\end{abstract}

\section{INTRODUCTION}

Quantum cryptography or quantum key distribution (QKD) has known an increasing interest because it offers higher security than public-key based key transfer systems [1]. Several systems [1-4] have been developed to exchange quantum keys via optical fibres, achieving key distribution up to $100 \mathrm{~km}$ [5]. In a recent experiment [6], a $23 \mathrm{~km}$ key transmission was performed in free space, hence demonstrating the feasibility of QKD for ground-to-ground or space application. However, only one method based on polarization-coded quantum states has been explored to realize free-space key distribution.

We report a new free-space transmission quantum key distribution method, using a direct-modulation technique associated to a single sideband detection method [7]. The use of directly modulated laser diodes and standard electronical components at the emitter enables suitable integration and thus offers potential 
satellite-to-ground applications. The experimental prototype operates at $850 \mathrm{~nm}$ using off-the-shelf components.

\section{PRINCIPLE}

Figure 1 shows the proposed system.

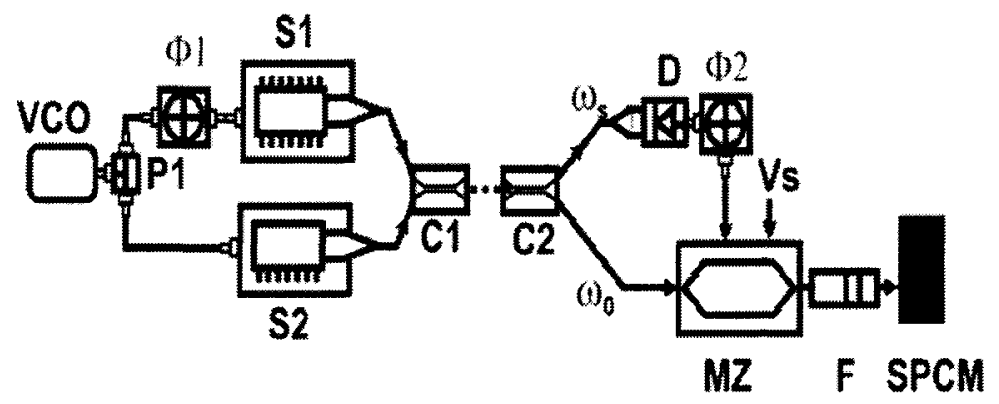

Figure 1. Schematic diagram of the direct modulation scheme.

The source (S1) is an attenuated laser diode operating at optical frequency $\omega_{0}$ (quantum signal). $\mathrm{S} 1$ is directly modulated at $\Omega<\omega_{0}$ with a modulation depth $m<<1$. The modulating signal is produced by a voltage controlled oscillator (VCO) that drives simultaneously a second laser diode S2 operating at optical frequency $\omega_{s}$. Both optical signals are transmitted thanks to a WDM coupler (C1). Their optical spectra are composed of a central peak and two sidebands at frequencies $\omega_{0} \pm \Omega\left(\omega_{s} \pm \Omega\right)$ with phase $\Phi_{l}(0)$ relative to the central peak. The phase $\Phi_{1}$ is introduced by a phase shifter. At the receiver, a WDM demultiplexer (C2) separates the transmitted signals. The synchronisation signal is converted by a detector (D) that generates an electrical signal at frequency $\Omega$. The amplitude of the electrical signal is matched to the emitter modulation depth $m$ and drives an unbalanced integrated Mach-Zehnder modulator (MZ). An additional phase shift $\Phi_{2}$ is introduced thanks to a second phase shifter. The bias voltage Vs is matched to the chirp value of the source $\mathrm{S} 1$ such that the probability $P_{1}$ and $P_{2}$ of detecting one photon in the lower and the upper sidebands of the quantum signal are respectively governed by a sine-squared and a cosine-squared function of the phase difference $\left(\Phi_{1}-\Phi_{2}\right)$. The sidebands are separated by an optical filter $(F)$ and photons are counted by a single photon counting module (SPCM). The BB84 protocol can then be implemented with only one detector as shown in [8]. 


\section{EXPERIMENT}

The experimentals circuits are shown in figure 2 and 3 . The actual prototype size emitter (fig.2) is $15 \times 10 \mathrm{~cm}$.The quantum signal is generated by a $1 \mathrm{~mW}$, $852 \mathrm{~nm}$ DBR laser diode. The modulation amplitude is set to $m \approx 0.3 \mathrm{~V}$ thanks to an integrated VCO and amplifier (RF circuit). Calibrated attenuators attenuate the light so that with the chosen value of $m$, the average photon number sent in the fibre is approximately 0.21 photon per pulse in each modulation sideband. The operating frequency is $2 \mathrm{GHz}$. The synchronisation signal at $2 \mathrm{GHz}$ is produced by the same VCO and modulates a standard CATV DFB laser (DMDFB) emitting at $1310 \mathrm{~nm}$.

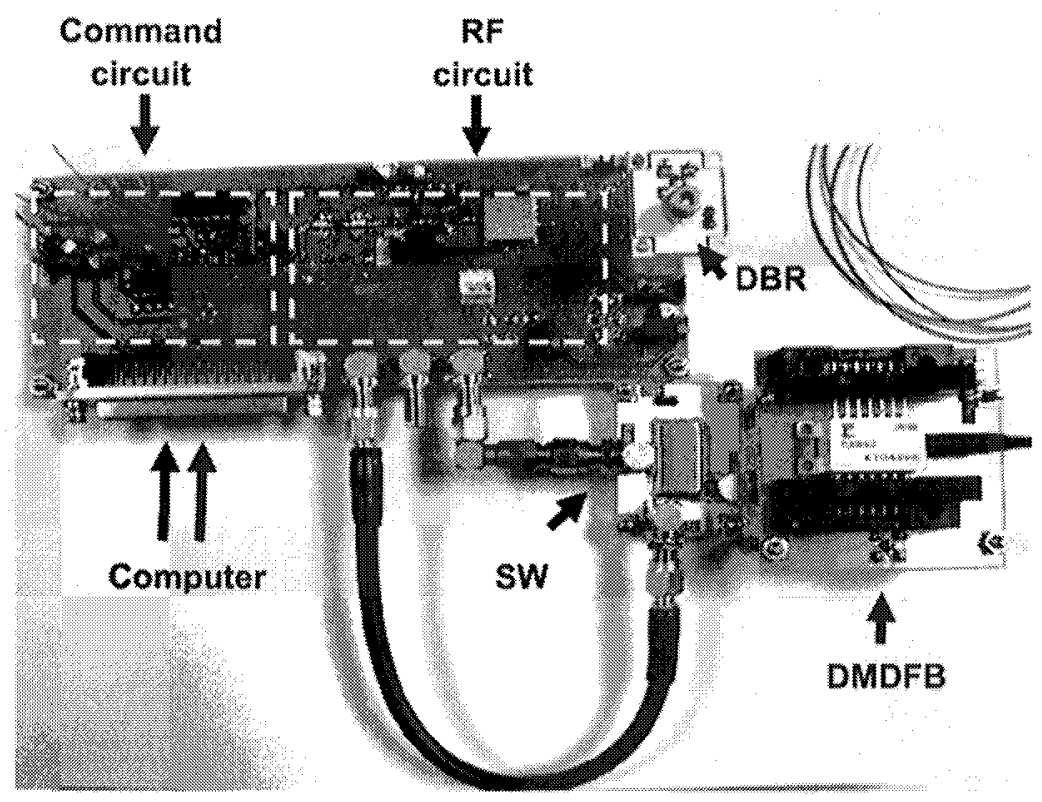

Figure 2. Prototype of the emitter.

Its average power was set to $10 \mu \mathrm{W}$ to avoid crosstalk with the quantum signal. At the emitter a computer controls a quadrature phase-shift-keying (QPSK) modulator (including in the RF circuit) to introduce a phase variation $\Phi_{I}$, randomly chosen among four possible values. An additional square-modulation at $1 \mathrm{MHz}$ is mixed with the synchronization signal to be used as a clock thanks to an external RF switch (SW). The command circuit converts the digital signal generated by the computer into the required analog signal. 


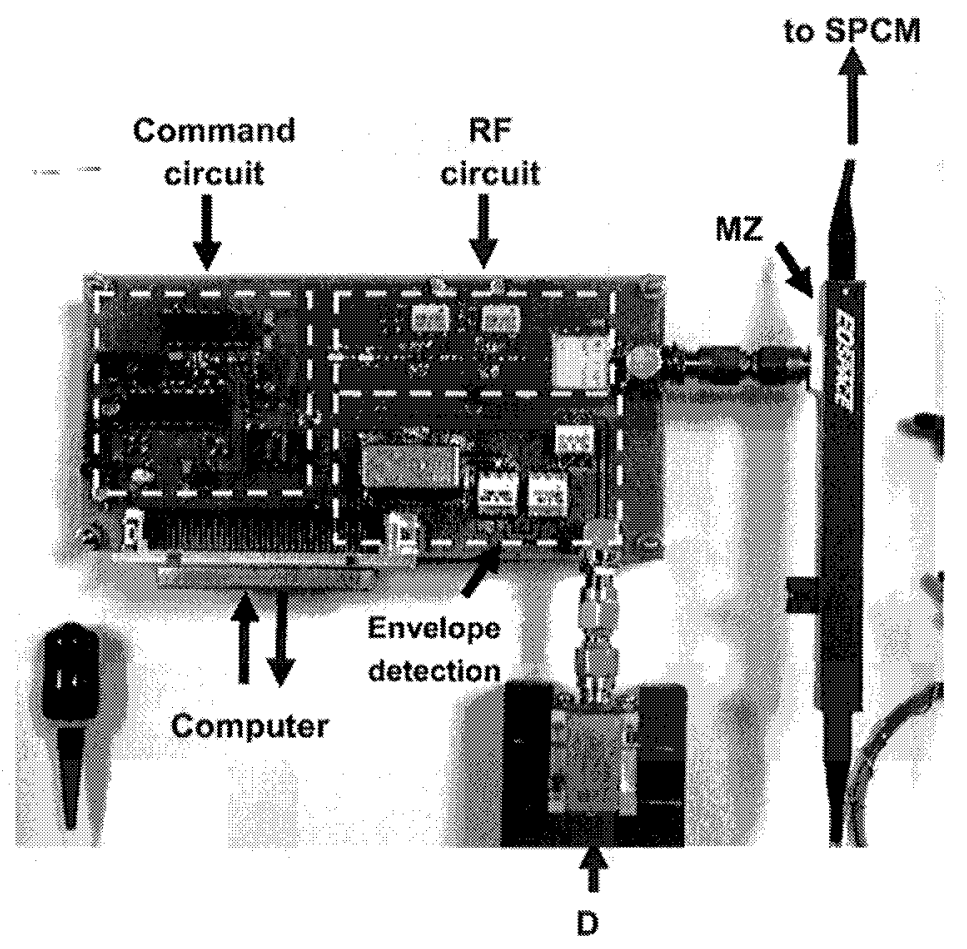

Figure 3. Prototype of the receiver.

At the receiver (fig.3), the quantum and the synchronization signal are separated by a $30 \mathrm{~dB}$ isolating WDM demultiplexer (not shown in fig 3 ). The synchronisation signal is first converted into an electrical signal by a standard detector (D). The resulting electrical signal is then split and filtered in two different ways to obtain in one case the plain $2 \mathrm{GHz}$ frequency and in the other case the $1 \mathrm{MHz}$ square envelope. This $1 \mathrm{MHz}$ clock is used to switch randomly and synchrounously the phase $\Phi_{2}$ among four possible values and generates a $50 \mathrm{~ns}$ duration gate that allows the EGG single photon counting module to be gated. In these conditions the quantum efficiency and the dark count per gate are $50 \%$ and $1.10^{-6}$. We use a customized $\mathrm{LiNbO}_{3}$ intensity modulator (MZ) and fibre FabryPérot interferometer (composed of two bragg gratings, not shown in fig 3) with a FSR of $10 \mathrm{GHz}$ and a finesse of 100 to select only one sideband. Quantum signal was detected by a SPCM. Key distributions have been performed with a $100 \mathrm{~m}$ single mode fibre at $850 \mathrm{~nm}$. The reconciliation process $[9,10]$ uses a LAN connection as the public channel. The global attenuation of the receiver was around 
$6 \mathrm{~dB}$ at $850 \mathrm{~nm}$. The quantum bit error rate (QBER) measured was thus found to be around $1 \%$ for a raw bit rate of $5 \mathrm{Kc} / \mathrm{s}$.

\section{CONCLUSION}

We have reported a new direct modulation method using a SSB detection scheme. Unlike a recent free space polarization based QKD experiment [11], the synchronisation and quantum signal used the same transmission channel. The first results obtained show the possible used of our method in free space transmission. Finally, the laser diodes can be replaced by VCSEL sources allowing future onchip integration of the emitter with hybrid CMOS VLSI [12] electronic circuits. This work is under progress.

\section{ACKNOWLEDGMENTS}

I wish to thank Samuel Moec for his invaluable help during the design of electronic circuits. This work was supported by FRANCE TELECOM under contrat $\mathrm{N}^{\circ}$ : $991 \mathrm{~B} 489$ and is protected under patent number : WO 02/049267 A1.

\section{REFERENCES}

[1] C.H.Bennett, F.Bessette, G.Brassard, L.Salvail and J.Smolin, "Experimental quantum cryptography", Journal of Cryptology, 5 (1992), 3.

[2] A. Muller, H. Zbinden and N. Gisin, "Quantum cryptography over $23 \mathrm{~km}$ in installed under-lake telecom fibre", Europhysics Letters,33 (1996), 335-339.

[3] P.D. Townsend, J.G. Rarity and P.R. Tapster, "Single photon interference in 10 km long optical fibre interferometer", Electronics Letters, 29 (1993), 634-635.

[4] R.J. Hughes, G.L. Morgan and C.G. Peterson, "Quantum key distribution over a 48km optical fiber network," Journal of Modern Optics, 47 (2000), 533-547.

[5] H.Kosaka, A.Tomita, Y.Nambu, T.Kimura and K.Nakamura, "Single-photon interference experiment over $100 \mathrm{Km}$ for quantum cryptography system using balanced gated-mode photon detector", Electronics letters, 39 (2003), 1199-1201.

[6] C. Kurtsiefer, P. Zarda, M. Halder, P.M. Gorman, P.R. Tapster, J.G. Rarity and H. Weinfurter, "Long distance free-space quantum cryptography", Proceedings of the SPIE, 4917 (2002), 25-31.

[7] O.L. Guerreau, J.-M. Merolla, A. Soujaeff, F. Patois, J.-P. Goedgebeur, F.J. Malassenet, "Long-distance QKD transmission using single-sideband detection scheme With WDM synchronization", Journal of Selected Topics in Quantum Electronics, 9 (2003), 1533-1540. 
[8] P. Moller, C. Schori, J.L. Sorensen, L. Salvail, I. Damgard and E. Polzik, “ Experimental quantum key distribution with proven security against realistic attacks", Journal of Modern Optics, 48 (2001), 1921-1942.

[9] G. Gilbert and M. Hamrick, "Secrecy, Computational Loads and Rates in Practical Quantum Cryptography", Algorithmica, 34 (2002), 314.

[10] D. Gottesman and H.-K. Lo, "Proof of security of quantum key distribution with twoway classical communications", IEEE Transactions on Information Theory, 49 (2003), 457.

[11] J.C. Bienfang, A.J. Gross, A. Mink, B.J. Hershman, A. Nakassis, X.Tang, R.Lu, D.H. Su, C.W.Clark, C.J. Williams, E.W. Hagley, J. Wen "Quantum key distribution with 1.25 Gbps clock synchronization", Optics Express, 12 (2004), 2011-2016.

[12] A.V. Krishnamoorthy and D.A.B. Miller, "Scaling optoelectronic-VLSI circuit into the 21st century: a technologiy roadmap" , Journal of Selected Topics in Quantum Electronics, 2 (1996), 55-76. 